\section{¿QUÉ EVALÚAN LOS INSTRUMENTOS DE EVALUACIÓN? VALORACIONES DE ESTUDIANTES}

\author{
Mónica Porto Currás \\ María Luisa García Hernández \\ Elisa Navarro Medina \\ Departamento de Didáctica y Organización Escolar, \\ Facultad de Educación - Universidad de Murcia \\ Campus Universitario de Espinardo, Murcia, España \\ monicapc@um.es; \\ luisagarcia@um.es; \\ elisanavarro@um.es
}

\begin{abstract}
Cómo citar este artículo/ Citation: Porto Currás, M.; García Hernández, M. L. y Navarro Medina, E. (2013). ¿Qué evalúan los instrumentos de evaluación? Valoraciones de estudiantes. Arbor, 189(760):a018. doi: http://dx.doi.org/10.3989/ arbor.2013.760n2004
\end{abstract}

Recibido: 18 marzo 2011; Aceptado: 3 octubre 2011.

RESUMEN: Los instrumentos empleados para la recogida de información aportan la información para evaluar si se han logrado las competencias previstas en un plan formativo. Por ello, conocer cómo entienden y describen los estudiantes las capacidades y destrezas que están demostrando en ellos, es una información a tener en cuenta en momentos de cambio y reestructuraciones de los planes de estudio.

En este artículo mostramos los resultados obtenidos en una investigación, en la que los estudiantes describen qué implica cada una de las técnicas de evaluación que han realizado a lo largo de sus estudios de licenciatura y qué destrezas debían emplear en cada una de ellas. Con la información recogida, hemos intentado organizar las tipologías de instrumentos de recogida de información con las capacidades desarrolladas desde las percepciones de los estudiantes, presentando un continuo de habilidades cognitivas.

PALABRAS CLAVE: Evaluación; evaluación universitaria; evaluación de aprendizajes; técnicas de evaluación; voces de estudiantes; capacidades cognitivas.

\section{WHAT DO EVALUATION TOOLS EVALUATE? STUDENTS' ASSESSMENTS}

Copyright: ㄷ 2013 CSIC. Este es un artículo de acceso abierto distribuido bajo los términos de la licencia Creative Commons Attribution-Non Commercial (by-nc) Spain 3.0.

ABSTRACT: The instruments used for information gathering provide the information to assess whether students have achieved the competences set out in the training plan. Therefore, knowing how they understand and describe the capabilities and skills they are demonstrating is useful information to be considered at times of change and curriculum restructuring.

In this paper we describe the results of study in which students describe what is involved in each of the assessment techniques they have undergone during their undergraduate studies and what skills were used in each case. With this information, we have tried to organise the types of tools to collect information with skills developed from the perceptions of students, presenting a continuum of cognitive abilities.

KEYWORDS: Assessment; university evaluation; assessment of learning; evaluation techniques; student voices; cognitive abilities. 


\section{INTRODUCCIÓN}

Es ampliamente aceptado que diferentes instrumentos sirven para valorar diferentes aprendizajes (Velilla, 1979; Rodríguez Diéguez, 1980; Zabalza, 1991, 2003; Biggs, 2006; Brown y Glasner, 2007; Hernández Castilla y Salinas, 2008). De esta forma, podemos encontrar diferentes clasificaciones sobre las capacidades, destrezas o conocimientos que permiten valorar cada herramienta e instrumento de evaluación, e incluso sobre la "validez" de la información que proporciona cada técnica.

Consecuentemente, la elección de una determinada técnica o de un conjunto de ellas estará en función de los objetivos y de las concepciones que tenga el profesor sobre el proceso de enseñanza-aprendizaje, "por ejemplo, si uno de unos objetivos ha sido que aprendan a expresarse correctamente y con coherencia, difícilmente lo podremos comprobar mediante una prueba de tipo test de elección múltiple. Será fácil de corregir, podrá recoger información sobre la comprensión de un texto, pero no sobre la capacidad del alumno para expresarse con corrección y coherencia" (Hernández y Salinas, 2008, 243).

Sin embargo, son menos frecuentes las investigaciones que se centran en analizar qué dicen los estudiantes sobre las capacidades, destrezas o aprendizajes que desarrollan en función del instrumento de evaluación que utilicen sus profesores. Y todo ello a pesar de haber un gran acuerdo en considerar que los procedimientos que se emplean para evaluar condicionan en gran medida lo que los estudiantes aprenden (Porto Currás, 2006, 2009), al mismo tiempo que se defiende que la metacognición sobre lo que se está aprendiendo es una de las principales formas de poder conseguir la autorregulación del propio aprendizaje.

Así pues, este artículo tiene como objeto principal analizar cómo describen los estudiantes las diferentes respuestas cognitivas que demandan los instrumentos o técnicas que los docentes emplean para recoger información. Para ello nos hemos basado en una investigación que tenía como finalidad ofrecer una radiografía de las técnicas de evaluación que emplean los docentes para obtener información del aprendizaje de sus estudiantes, en una titulación concreta, en este caso Pedagogía, y en la promoción 2002-2007. No obstante, hemos de tomar los resultados que a continuación mostramos con cautela, ya que la intención principal de la investigación no era la de recoger información sobre las capacidades que desarrollaban los estudiantes, sino sobre las técnicas de evaluación, por lo que no se le realizó ninguna pregunta específica sobre esta cuestión. Aún así, las descripciones y ejemplificaciones obtenidas nos han permitido aproximarnos a la visión que el alumnado tiene sobre las demandas cognitivas solicitadas por cada instrumento.
Concretamente, los datos empíricos proceden de una tesis de Licenciatura, titulada: "Los instrumentos de evaluación en la Licenciatura de Pedagogía de la UMU (2002-2007)", en la que se obtuvieron 973 descripciones $^{2}$ de lo que los estudiantes describían como herramientas de evaluación. En estas evocaciones, los alumnos han identificado hasta 25 modalidades distintas de técnicas para la recogida de información sobre su aprendizaje; tipologías que hemos organizado en torno a cuatro grandes referentes: exámenes orales, exámenes tipo test, exámenes escritos y trabajos, de acuerdo con la categorización que ellos mismos propusieron.

\section{METOdOLOGÍA DE LA INVESTIGACIÓN}

Hemos intentado acercarnos a las experiencias de los discentes, por lo que hemos optado por una metodología que combine distintas técnicas, que se sitúan dentro de los denominados métodos descriptivos (Salkind, 1999). Así pues, tratamos de conocer una parte de la realidad que sucede en las aulas, para luego poder extraer conclusiones y proporcionar propuestas de mejora.

Dada la naturaleza de la información buscada, era necesaria una metodología de integración cualitativa - cuantitativa para comprender mejor el ámbito de estudio. De acuerdo con Greene y Everston (1989), consideramos que la combinación de ambas metodologías, en un mismo estudio, es la forma más adecuada de tratar los datos en las investigaciones en Ciencias Sociales. Así pues, tras revisar algunas técnicas para obtener información (Delgado y Gutiérrez, 1994; Martínez, 1998), entre las que destacamos los cuestionarios, las entrevistas a docentes y discentes, la observación de clases, el relato de los alumnos, la estimulación del recuerdo y los grupos de discusión; nos ha parecido acertado emplear en esta investigación: el recuerdo a través de materiales, (estimulando así el recuerdo en pequeño grupo) y las entrevistas, que nos han permitido contrastar la información obtenida anteriormente.

Más concretamente, el primero de los instrumentos, el recuerdo a través de materiales, consistió en proporcionar a los estudiantes un documento donde aparecían las asignaturas ofertadas en la titulación de Pedagogía en la promoción 2002-2007, y por otro lado, otro documento con una categorización de las diversas técnicas de evaluación ${ }^{3}$. Así, los discentes mediante el recuerdo en pequeño grupo, fueron identificando y clasificando cada instrumento en cada una de las asignaturas de acuerdo con los 4 grandes referentes: trabajos, exámenes escritos, exámenes tipo test y exámenes orales.

Una vez cumplimentado este primer documento se les solicitó a los estudiantes que además de clasificar, 
intentasen recordar qué significaba para ellos cada tipo de instrumento de evaluación, para posteriormente describirlo y ejemplificarlo. Así, los alumnos procedieron a relatar qué entendían por trabajos, exámenes escritos, tipo test y por pruebas orales.

Por otra parte la segunda técnica, la entrevista, fue llevada a cabo tras la puesta en práctica del recuerdo a través de materiales y tenía como finalidad solventar algunas controversias surgidas en el recuerdo en pequeño grupo. Es de resaltar, por tanto, que el objeto de estas entrevistas no era recoger nueva información, sino aclarar cuestiones sobre las que aparecía ambigüedad o confusión tras el análisis de los materiales. Asimismo, el carácter argumentativo de las respuestas, durante la entrevista, complementaron y enriquecieron cualitativamente los resultados. En resumen, podemos señalar que los instrumentos empleados para la recogida de información nos han permitido aproximarnos a la evaluación del aprendizaje que se había llevado a cabo a lo largo de estos cinco años de carrera, desde la perspectiva del alumno.

Nuestra muestra de estudio quedó conformada por 103 estudiantes que cursaban 5o de Pedagogía en 2007 y que, consecuentemente, pertenecían a la promoción que se analizó (2002-2007).

Cabe destacar que el número total de alumnos matriculados en esta promoción en las asignaturas troncales y obligatorias ${ }^{4}$ era de 125 estudiantes, lo que supone que hemos llegado al $82,4 \%$ del total de los discentes matriculados. Además, podemos corroborar que tenemos información de todas las asignaturas cursadas ${ }^{5}$, independientemente de su naturaleza y que se ha llegado a un número representativo de alumnos.

Los 103 discentes fueron reorganizados en 34 grupos compuestos por 3 alumnos, de los cuales se recogía la información. Se tomó la determinación de organizar en grupos de 3 personas puesto que favorecía el debate y la rememoración de detalles que de forma individual hubiesen sido muy difíciles de recuperar.

En cuanto a los programas utilizados para el tratamiento de los datos obtenidos, se ha utilizado la base de datos Microsoft Excel, una herramienta potente de tratamiento de datos y análisis estadístico; y el programa Nudist, una herramienta diseñada para manejar datos no numéricos y no estructurados en análisis cualitativos, soportar procesos de codificación de datos en un sistema de catalogación, búsquedas de texto o modelos de código y teorizar sobre los datos. El método seguido para introducir la información fue la trascripción de todas las descripciones de los estudiantes para posteriormente crear categorías en las que organizar los datos y así facilitar un análisis de los resultados obtenidos.
El programa Excel nos permitió introducir las 973 descripciones, teniendo con ello una visión general de toda la información, codificando los datos, estableciendo las categorías, organizando todos los resultados, ordenando la información a través de tablas y comparando los distintos resultados. Mientras que el programa Nudist fue utilizado específicamente para el análisis vivencial, ya que es una de sus funciones primordiales. Concretamente se empleó tanto para las descripciones como para la información extraída de las entrevistas.

\section{RESULTADOS}

Los estudiantes de Pedagogía de la promoción 20022007 han señalado como técnicas de evaluación: los trabajos, los exámenes escritos, los exámenes tipo test y los exámenes orales. Sin embargo, dentro de estas grandes tipologías los discentes han identificado más de 25 modalidades de herramientas de recogida de información.

Los trabajos han sido, según los estudiantes, la herramienta más empleada por los profesores para obtener información (90,3\%).

Teniendo presente la clasificación que propone Nieto y Vallejo (2010) y a través de las descripciones de los discentes hemos optado por establecer dos niveles: los trabajos de apoyo y los trabajos de formación. Más concretamente, en los trabajos de apoyo hemos agrupado: la búsqueda de información, la elaboración de memorias y el uso de las nuevas tecnologías. Como trabajos de formación hemos considerado: trabajos de revisión, reflexión, inicio al diseño e inicio a la investigación (tabla 1 ).

Dentro de los trabajos de apoyo, se encuentran los trabajos de búsqueda de información. Los estudiantes afirmaban que este tipo de trabajo se utilizaba para: profundizar en determinados temas, mejorar las habilidades en el uso de bases, enviar la información por correo, y esto por su facilidad de acceso y gran disposición de fuentes para reunir la información. Más concretamente, los alumnos rememoraban que estos trabajos se realizaban para:

- GO28... adjuntar todos aquellos enlaces de páginas Web...

.G06... complementar...

- G013... mejorar nuestras habilidades...

Otro de los trabajos, incluidos en este primer nivel, son las memorias de prácticum y las memorias de experiencias, siendo conscientes de qué se debía de hacer en cada modalidad: cuando hablamos de memorias de prácticum consiste en realizar un informe y cuando se trata de memorias de experiencias incluyen una reflexión y/o valoración sobre lo que se ha aprendido y cómo. 
Tabla 1. Tipología de trabajos

\begin{tabular}{|c|c|c|c|}
\hline \multicolumn{2}{|r|}{ Trabajos de Formación } & \multicolumn{2}{|r|}{ Trabajos de Apoyo } \\
\hline Revisión & $\begin{array}{l}\text { Se realiza una lectura comprensiva, de textos, } \\
\text { libros, charlas, etc., para posteriormente } \\
\text { extraer las ideas principales y realizar un } \\
\text { mapa conceptual, un resumen, una síntesis, } \\
\text { etc., con la finalidad de profundizar y conocer } \\
\text { más acerca de un tema relacionado con la } \\
\text { asignatura. }\end{array}$ & $\begin{array}{l}\text { Búsqueda de } \\
\text { información }\end{array}$ & $\begin{array}{l}\text { Aquellos en los que mediante } \\
\text { Internet o en materiales escritos } \\
\text { (libros, revistas, textos, artículos, } \\
\text { etc.), consultaban información, } \\
\text { sobre un determinado tema. }\end{array}$ \\
\hline Reflexión & $\begin{array}{l}\text { Lectura comprensiva de un material escrito } \\
\text { (libro o texto) para posteriormente realizar } \\
\text { una valoración, una crítica constructiva o } \\
\text { reflexionar sobre él. }\end{array}$ & $\begin{array}{c}\text { Elaboración de } \\
\text { memoria }\end{array}$ & $\begin{array}{l}\text { Redacción de los aspectos más } \\
\text { importantes a nuestro criterio } \\
\text { y elaboración de un informe } \\
\text { donde reflejar todas las acciones } \\
\text { diarias, resúmenes, mapas } \\
\text { conceptuales y prácticas llevadas } \\
\text { a cabo, incluyendo una reflexión } \\
\text { y/o valoración sobre lo que se ha } \\
\text { aprendido y cómo. }\end{array}$ \\
\hline Diseño & $\begin{array}{l}\text { El alumno sepa ha de aplicar el conocimiento } \\
\text { a situaciones particulares, teniendo como } \\
\text { resultado un proyecto o programa. }\end{array}$ & $\begin{array}{l}\text { Uso de nuevas } \\
\text { tecnologías }\end{array}$ & $\begin{array}{l}\text { Consiste en el uso, aprendizaje } \\
\text { y dominio de diferentes } \\
\text { herramientas telemáticas. }\end{array}$ \\
\hline Investigación & $\begin{array}{l}\text { Trabajo deaplicación con carácterinvestigador, } \\
\text { en el que el estudiante debe de partir de } \\
\text { una hipótesis que requiere la respuesta en } \\
\text { términos de nuevo conocimiento, además se } \\
\text { le exige que recoja y analice datos. }\end{array}$ & & \\
\hline
\end{tabular}

Sin duda, es en las memorias de experiencias donde podemos encontrar un punto de inflexión entre las destrezas llevadas a cabo por los estudiantes, que pueden ir desde las más simples a las más complejas.

Así, hemos optado por distinguir dos bloques: uno primero en el que se encuentran aquellos trabajos que tienen una estructura general, normalmente hacen referencia a una actividad realizada en un momento concreto, y que habitualmente lo describen como un documento (prácticas que se han ido realizado y entregando al profesor en horas de clase, en las que había que relacionar el contenido). Y, por otro lado, un segundo bloque donde encontramos lo que el discente califica como diario y portafolio. La distinción entre ambos términos radica en que el diario conlleva una descripción constante y marcada por lo que se sucede en cada momento (recopilación ordenada por fechas de todo lo que se hace día a día en clase), mientras que en el portafolio no es tanto lo que ocurre en cada momento, sino qué y cómo se ha aprendido (documento que recoge: resúmenes, mapas conceptuales, prácticas y una reflexión lo que ha aprendido y como y finaliza con una valoración personal). Por tanto, hemos establecido la línea divisoria entre diario y portafolio en que en el primero no aparece una reflexión profunda sobre su propio aprendizaje, mientras que en el portafolio se comienzan a dar pinceladas de esta consideración.
Centrándonos ahora en los trabajos de formación, Nieto y Vallejo (2010) acuñan al término trabajos de revisión, señalando que: este tipo de trabajo propone a los estudiantes actividades de conocimiento declarativo sobre un tema. La finalidad es desarrollar el conocimiento de la materia en diferentes grados de profundidad. Es significativo cómo los estudiantes a través de sus descripciones identifican una tipología de trabajo que se asemeja considerablemente a la descrita por estos autores, de esta manera los alumnos relatan que estos trabajos incluían:

$$
\begin{array}{ll}
\text { - G034... } & \text { elaboración de un resumen... } \\
\text { - G015... } & \text { para realizar una síntesis... } \\
\text { - G08... } & \text { elaboración de un mapa extrayendo } \\
\text { las ideas principales... }
\end{array}
$$

- GO7... lectura comprensiva de los temas...

- GO26... lectura de unos textos... para extraer las ideas principales...

- GO24... lectura de textos para luego trabajar sobre ellos en un debate.

- G014... tras la lectura de diversos textos se tenía que realizar un comentario...

\footnotetext{
- GO2... p profundización sobre el tema...

- G011... tras la asistencia a una charla se realizaba un comentario sobre la misma...
} 
- G014... se trataba de debatir o comentar algún tema de interés social actual que estaba relacionado con la política...

- G01... nos referimos a la comparación de dos temas, ver en qué se parecen y en que se diferencias dos temas o aspectos.

-GO7... requiere ampliar y profundizar acerca de un tema o información concreto.

\section{- GO27... el profesor plantea un tema o una serie de temas y cada uno elegía uno sobre el que profundizaba}

Como podemos comprobar, las descripciones que los estudiantes hacen del tipo de actividades incluidas en los trabajos de revisión puede ir desde la recensión, el resumen, la elaboración de mapas conceptuales, hasta la realización de comentarios, comparaciones y profundizaciones en determinados aspectos de la materia.

En cuanto a los trabajos de reflexión, Nieto y Vallejo (2010) los definen como: un trabajo que exige la revisión y comprensión de un material para poder realizar deducciones y emitir juicios de valor. El conocimiento, en este caso, es interpretativo no solamente declarativo y, a diferencia del anterior incorpora un análisis de valor. Los estudiantes, al igual que con los trabajos de revisión, han realizado una serie de relatos de los cuales hemos extraído las siguientes descripciones:

. G031... lectura y valoración de varios libros...

- G013... tras la lectura realizar una crítica...

- G04... crítica constructiva de los textos...

- G07... requería una valoración...

. G010... Reflexión sobre los textos...

· GO22... reflexión sobre el libro...
Por tanto, a través de las descripciones de los alumnos, se expresan qué capacidades han desarrollado para los trabajos de reflexión y qué tareas debían realizar: tareas de opinión y valoración:

- G01... nos referimos a la lectura de un libro, posteriormente realizar un comentario personal donde tenemos que dar nuestra opinión personal de los temas tratados en el libro.

- G026... lectura de un libro, debíamos realizar un comentario de su contenido y posteriormente expresar nuestra opinión...

- GO5... Se realiza una lectura y valoración personal sobre libros.

- G021...se nos pedía la lectura comprensiva de un libro y una valoración personal

- G012...lectura, argumentación y valoración de libros...

Teniendo en cuenta todas las descripciones relativas a los trabajos de revisión y reflexión que realizan los alumnos, debemos concluir que estos trabajos constituyen un continuo en relación a las capacidades que solicitan a los estudiantes, tal y como sintetizamos en el siguiente cuadro (cuadro $\mathrm{n}$ ㅇ 1).

De la misma forma, los estudiantes a través de sus descripciones relataban que los trabajos de inicio al diseño implicaban: la realización de un informe $o$ diagnóstico e incluían los resultados de una serie de pruebas realizadas al sujeto con sus conclusiones $y$ orientaciones. También puede darse el caso de diseñarse intervenciones donde aparece una planificación de los objetivos, recursos y actividades enfocados a la mejora de las necesidades del sujeto y/o situación analizada. Algunos ejemplos de sus evocaciones serían:

Figura 1. Capacidades desarrolladas para los trabajos de revisión y reflexión

Trabajos de REVISION

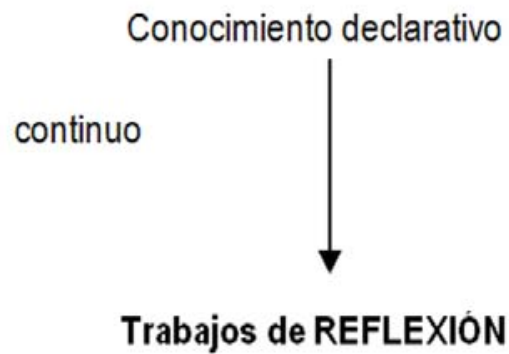

Conocimiento interpretativo
Desarrollandolas capacidades de:

\section{COMPRENSION}

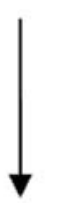

VALORACIÓN 
- G07... realización de un informe o diagnóstico ...

. GO21... donde se recogen orientaciones... y se realiza tras la aplicación de pruebas...

- G01... realización de un proyecto de intervención, donde teníamos que planificar los objetivos, recursos, actividades...

- G015 ... en un contexto determinado realizar una intervención y unas orientaciones para la mejora...

Estas descripciones coinciden en gran medida con la definición que Nieto y Vallejo realizan de los trabajos de inicio al diseño, en los que se considera que el alumno debe saber desarrollar y aplicar el conocimiento a situaciones particulares, obteniendo como resultado un proyecto o programa. Tal y como señalan estos autores en su definición, éste tipo de trabajos tiene como meta no sólo que el discente entienda el contenido, sino que sea capaz de planificarse, aplicarlo, valorarlo y ofrecer propuestas de mejora; capacidades que los alumnos reconocen en la realización de estos trabajos.

Por otra parte, en cuanto a los trabajos de inicio a la investigación, de acuerdo con la propuesta de Nieto y Vallejo destacamos que son un trabajo de aplicación de carácter investigador, donde el estudiante debe de partir de una hipótesis que requiere la respuesta en términos de nuevo conocimiento, además se le exige que recoja y analice datos. De nuevo encontramos una gran coincidencia en los relatos de los alumnos, en los cuales identificaban que los trabajos de inicio a la investigación son aquellos en los que el estudiante parte de una hipótesis, para ello busca información de forma exhaustiva, indaga, obtiene datos, los ordena, sintetiza y analiza. Y a partir de ahí plantea los objetivos y la metodología (cuantitativa o cualitativa) para extraer conclusiones y comprobar si se cumple o no la hipótesis, incluyendo en ocasiones información innovadora.

- G010 ... debíamos buscar información sobre un tema, y a partir de ahí plantear objetivos, metodología ...

- GO33 ... profundizar sobre un tema vigente y actual referido a la asignatura, llevando a cabo una investigación sobre los diferentes aspectos de esa problemática ...

- G011 ... recoger información utilizando diversos instrumentos, analizar datos, extraer conclusiones, comprobar si se cumple o no la hipótesis ...

- GO22 ... recabar información sobre un tema, datos estadísticos, estudios, etc., analizar, seleccionar y sacar conclusiones ...

Más concretamente, en este tipo de trabajos los alumnos ponen en práctica habilidades de distinta complejidad, desde las más simples: como la búsque- da de información, organización de ésta, hasta las más complejas como: la indagación, formulación de hipótesis y el análisis y la extracción de conclusiones.

Consecuentemente, los trabajos de inicio al diseño e investigación también nos muestran un continuo, en el que se requiere de los estudiantes que analicen una situación (real o supuesta), informando de sus circunstancias para poder orientar su intervención, o bien investigarla para proponer nuevas actuaciones.

En cuanto a los exámenes escritos han sido aplicados en el $56 \%$ de las disciplinas. La muestra de este estudio identifica a través de sus descripciones 4 tipos de enunciados en estos exámenes, que requerían: respuesta tipo ensayo, respuesta corta, respuesta sobre materiales escritos, y resolución de ejercicios y casos prácticos.

Pero, a su vez, también diferencian las destrezas desarrolladas para cada uno de estos enunciados, que van desde las más simples (reconocimiento-recuerdo) hasta las más complejas (análisis y utilización del conocimiento). Cabe señalar que cada proceso cognitivo está compuesto por todos los anteriores, siendo esto un dato fundamental para comprender que la utilización del conocimiento conlleva recuperar la información, comprenderla y analizarla. Así pues, basándonos en los trabajos de Marzano (2007) y en los términos utilizados por los alumnos, se ha tipificado la información de la siguiente manera:

- Cuando los estudiantes hacen alusión a la memorización, lo atribuyen a recordar de forma explícita datos o informaciones almacenadas en la memoria. Podemos comprobar que esto estaría relacionado, de acuerdo con la clasificación de Marzano, con el primer proceso cognitivo: el de recuperación de la información (el recuerdo).

- De la misma forma, cuando los discentes relatan la relación entre contenidos, y dadas sus descripciones, las hemos contrastado con las que Marzano propone como comprensión, ya que no solamente recuerdan una información sino que además de identificarla, la sintetizan y la categorizan.

- Por último, los alumnos mencionan la capacidad de reflexionar. Para ello, describen que tenían de comprender bien el temario y justificar las respuestas. Así pues se ha optado por asociarlo a lo que Marzano categoriza como análisis puesto que en este caso los discentes además de recordar y comprender el conocimiento, lo aplican.

Podemos resaltar que los exámenes escritos que requieren una respuesta tipo ensayo o de respuesta breve, y que solicitan sólo la recuperación de la información, son los que se han empleado más frecuentemente (entre 30 y 40 asignaturas). De ellos, los discentes relatan que se resuelven a partir de la memo- 
rización de contenidos, ya que se pedía al alumno una respuesta lo más fiel posible al temario. No obstante, con esta frecuencia, también se han empleado los enunciados tipo ensayo que requieren el análisis de la información, de los cuales los estudiantes dicen que consistían en la realización de una prueba con preguntas muy generales, que para resolverlas es necesario comprender bien el temario, pues tienes que mostrar tu opinión o reflexión fundamentada o justificada.

Con una frecuencia también alta (entre 20 y 30 asignaturas) los alumnos afirman que también han tenido experiencias de exámenes con enunciados que requieren respuestas breves y que solicitan el análisis de la información (respuestas en base a unos contenidos trabajados a lo largo de la asignatura, pero no se trata de responder sobre contenidos concretos, sino que se valora la expresión, reflexión y relación entre conceptos); y exámenes que requieren una respuesta tipo ensayo, donde se solicitaba la comprensión de la información tratada (preguntas relacionando unos temas con otros, explicando bien porqué estaban relacionados).

Con esta misma frecuencia también relatan las experiencias con exámenes escritos que implicaban la resolución de ejercicios o casos prácticos, de los cuales recordaban que eran preguntas en las que debían de solucionarlas haciendo uso y poniendo en práctica la teoría aprendida, para dar solución a las actividades o a los supuestos. Consecuentemente, respecto a las habilidades puestas en práctica para solventar este tipo de preguntas los alumnos aludían a la aplicación del contenido.

Con una frecuencia menor (entre 10 y 20 asignaturas) se encuentras los exámenes con enunciados que requieren una respuesta breve y solicitan la comprensión de la información (conllevan la relación de diversos temas y conceptos para poder desarrollarlas satisfactoriamente); así como las preguntas sobre materiales escritos que demandan recuperación (las preguntas hacen referencia a un material escrito que había que memorizar) o análisis de la información (responder a una serie de cuestiones relacionadas con la lectura de diversos materiales escritos, en los que había que reflexionar de forma crítica).
En el otro extremo, encontramos como los menos empleados los exámenes escritos sobre materiales escritos que requerían por parte del alumnado la comprensión de la información (en menos de 10 asignaturas), de los que los alumnos describen que implicaban leer un libro de manera que entendiésemos su contenido o su relación con la teoría dada en clase.

En cuanto a los exámenes tipo test, han sido utilizados en el $44 \%$ de las asignaturas. Más concretamente, los alumnos han señalado que a lo largo de la carrera han realizado varias tipologías de test, entre las que se encuentran: los test de verdadero o falso, los test de elección única con tres o cuatro alternativas de respuesta y los test de elección múltiple.

Los discentes indicaban que las habilidades puestas en práctica para este tipo de pruebas eran la demostración del conocimiento del contenido y la discriminación de la respuesta correcta. A continuación recogemos algunas de sus descripciones:

- GO27... debíamos demostrar que sabíamos dichos conceptos y contenidos....

-G011... evalúan de esta forma los conocimientos adquiridos a lo largo del curso...

- G012... comprobar que los conocimientos son adquiridos y asentados...

- G01... tienes que demostrar tus conocimientos y comprensión de lo estudiado...

- G01... se hacen para ver que el alumno conoce realmente la respuesta...

- GO26... domináramos a la perfección los contenidos y las ideas que tenían que ver con el temario...

- G031... discriminación y selección de la respuesta correcta... una serie de ítems...debes identificar la respuestas correcta/ incorrecta a cada pregunta...

- GO27... discriminar las opciones, aunque sus cambios fueran mínimos...

- GO25... diferenciación y discriminación de conceptos...

Tabla 2. Capacidades desarrolladas en cada tipología de examen test

\begin{tabular}{|l|c|c|c|c|}
\hline & $\begin{array}{c}\text { Test Verdadero } \\
\text { / Falso }\end{array}$ & $\begin{array}{c}\text { Test 3 alternativas } \\
\text { de respuesta }\end{array}$ & $\begin{array}{c}\text { Test 4 alternativas de } \\
\text { respuesta }\end{array}$ & $\begin{array}{c}\text { Test de elección } \\
\text { múltiple }\end{array}$ \\
\hline Dominio de contenidos & $17,6 \%$ & $6,25 \%$ & $7,69 \%$ & $10,53 \%$ \\
\hline $\begin{array}{l}\text { Discriminar la respuesta } \\
\text { correcta }\end{array}$ & $23,5 \%$ & - & - & $2,63 \%$ \\
\hline
\end{tabular}


Pero como podemos comprobar en la tabla 2, estas demandas no se reconocen igual para todas las modalidades de exámenes tipo test, de modo que en los exámenes de respuesta única, con tres o cuatro alternativas de respuesta, los estudiantes no han mencionado la habilidad de discriminar la respuesta correcta del resto de ítems.

Por último, y respecto al examen oral, resaltar que ha sido el menos empleado en esta promoción de Pedagogía, solamente en 5 asignaturas (que suponen un 5,3\% del total); pero a pesar de ello los estudiantes tenían claro que con él ponían en práctica destrezas como: la fluidez, la organización mental del contenido y el control del lenguaje no verbal. Así los estudiantes recuerdan que:

- G015... para este tipo de evaluación hay que tener destrezas en la expresión y comunicación verbal, control sobre las emociones y nervios que suscita y claridad en las ideas a exponer para hablar de ellas con fluidez.

- G016... aplicando la habilidad de saber hablar en público,... teníamos que exponerlos teniendo en cuenta el lenguaje no verbal.

\section{CONCLUSIONES}

En primer lugar, indicar que hemos de tomar estos datos con cautela pues, como se anunciaba al inicio del artículo, la intención principal de la investigación no era la de recoger información sobre las capacidades que desarrollaban los estudiantes, sino sobre las técnicas de evaluación que se habían empleado en esta promoción de Pedagogía. De esta forma, hemos de ser conscientes de la necesidad de seguir profundizando sobre esta información en futuras investigaciones.

Pero con la información que los estudiantes nos han proporcionado, hemos de considerar que en el conjunto de las técnicas de evaluación se ha podido observar una evolución en cuanto a procesos cognitivos, puesto que se comienza con capacidades muy simples, como puede ser la rememoración hasta llegar a destrezas de orden superior, como es la aplicación de la teoría a la práctica. Así pues, hemos de resaltar que ha sido en muchas ocasiones un continuo en el que se ha realizado un recorrido por las distintas capacidades. En la tabla 3 hemos intentado organizar las tipologías de instrumentos de recogida de información con las capacidades desarrolladas, presentando este continuo de habilidades cognitivas desde las más simples (la discriminación de la respuesta correcta) hasta capacidades de orden superior (por ejemplo, valorar el propio aprendizaje o investigar sobre una situación educativa):

Tabla 3. Capacidades desarrolladas en cada instrumento de evaluación

\begin{tabular}{|c|c|c|c|c|c|}
\hline \multicolumn{6}{|c|}{ EXÁMENES TIPO TEST } \\
\hline \multicolumn{3}{|c|}{ Discriminar respuestas correctas de las erróneas } & \multicolumn{3}{|c|}{$\begin{array}{c}\text { Demostrar el dominio de los contenidos: conocer y } \\
\text { comprender }\end{array}$} \\
\hline \multicolumn{6}{|c|}{ EXÁMENES ESCRITOS } \\
\hline $\begin{array}{l}\text { Memorizar } \\
\text { contenidos }\end{array}$ & $\begin{array}{l}\text { Relacionar } \\
\text { temas }\end{array}$ & $\begin{array}{l}\text { Expresión, reflexión } \\
\text { y relación entre } \\
\text { conceptos }\end{array}$ & $\begin{array}{c}\text { Mostrar } \\
\text { opiniones o } \\
\text { reflexiones } \\
\text { fundamentadas } \\
\end{array}$ & \multicolumn{2}{|c|}{$\begin{array}{l}\text { Poner en práctica la teoría en } \\
\text { actividades o supuestos prácticos }\end{array}$} \\
\hline \multicolumn{6}{|c|}{ TRABAJOS } \\
\hline $\begin{array}{l}\text { Recoger y } \\
\text { ordenar las } \\
\text { actividades } \\
\text { realizadas en } \\
\text { el seno de una } \\
\quad \text { materia }\end{array}$ & $\begin{array}{l}\text { Complementar } \\
\text { información, } \\
\text { mediante la } \\
\text { búsqueda y } \\
\text { manejo de } \\
\text { fuentes de } \\
\text { información }\end{array}$ & $\begin{array}{l}\text { Resumir, comentar, } \\
\text { comparar o } \\
\text { profundizar sobre } \\
\text { determinados } \\
\text { aspectos de una } \\
\text { materia }\end{array}$ & $\begin{array}{l}\text { Comentar y } \\
\text { valorar textos } \\
\text { escritos }\end{array}$ & $\begin{array}{l}\text { Reflexionar y valorar } \\
\text { sobre lo aprendido } \\
\text { en una disciplina }\end{array}$ & $\begin{array}{l}\text { Analizar o } \\
\text { investigar sobre } \\
\text { una situación, } \\
\text { para orientar } \\
\text { su posible } \\
\text { intervención }\end{array}$ \\
\hline \multicolumn{6}{|c|}{ EXÁMENES ORALES } \\
\hline \multicolumn{6}{|c|}{$\begin{array}{l}\text { - Claridad en las ideas } \\
\text { - Destrezas en expresión y comunicación verbal } \\
\text { - Hablar con fluidez } \\
\text { - Hablar en público } \\
\text { - Control del lenguaje no verbal } \\
\text { - Control sobre las emociones y nervios }\end{array}$} \\
\hline
\end{tabular}


Como podemos comprobar, los alumnos afirman que son en los exámenes tipo test donde ponen en práctica destrezas más sencillas: discriminar y demostrar conocimiento marcando una respuesta; mientras que en los exámenes escritos y los trabajos se puede apreciar como aplican distintas destrezas en un creciente continuo de complejidad. Caso aparte son los exámenes orales, en los cuales los discentes destacan habilidades diferentes a las que ponen en práctica en las otras técnicas, cuestión fundamental a tener en cuenta, sobre todo considerando el escaso uso que se hace de esta herramienta de evaluación en la muestra estudiada.

Así pues, podemos constatar que los estudiantes tienen una imagen clara de cuáles eran las técnicas y qué debían de hacer en cada una de ellas. Sin duda, para ellos no se evalúa de igual forma con unas técnicas que con otras y esto queda reflejado en las aportaciones que elaboran sobre cada una de las herramientas (aún sin solicitarle esta información). No olvidemos que el qué y cómo aprenden los alumnos depende en gran medida de cómo se les evalúe.

Consecuentemente, si conocemos lo que los estudiantes valoran que se les solicita en cada instrumento de evaluación, podremos aproximarnos a conocer qué han aprendido o, por lo menos, qué destrezas consideran que han aprendido a lo largo de su proceso formativo en la Universidad. Además, sabemos que cuando evaluamos los aprendizajes que han realizado los alumnos, estamos también evaluando la enseñanza que hemos llevado a cabo. La evaluación nunca lo es, en sentido estricto, de la enseñanza o del aprendizaje, sino más bien, de los procesos de enseñanza y aprendizaje.
1 Tesina realizada por Mạ Luisa García Hernández, bajo la dirección de Nicolás Martínez Valcárcel y Mónica Porto Currás, y presentada en la Facultad de Educación de la Universidad de Murcia en noviembre de 2009 .

2973 descripciones son cada una de las aportaciones descritas por los estudiantes acerca de las técnicas de obtención de información.

\section{BIBLIOGRAFÍA}

Biggs, John (2006): Calidad del aprendizaje universitario. Madrid, Narcea.

Brown, Sally y Glasner, Ángela (2007): Evaluar en la universidad. Problemas y nuevos enfoques. Madrid, Narcea Ediciones.

Delgado, Juan Manuel y Gutiérrez, Juan (coords.) (1994): Métodos y técnicas cualitativas de investigación en Ciencias Sociales. Madrid, Síntesis Psicología.

García Hernández, María Luisa (2009): Los instrumentos de evaluación en la Licenciatura de Pedagogía de la UMU (20022007). Tesis de Licenciatura no publicada, Universidad de Murcia.

Greene, Judith y Everston, Carolyn. (1989): La observación como indagación y como método. En Wittrock, Merlin (comp.): La investigación de la enseñanza II. Métodos cualitativos y de observacion. Madrid, Paidós-MEC. 303-421.

Hernández Castilla, Reyes y Salinas, Bernardino (2008): La evaluación didáctica como componente del diseño/ desarro-
3 Esta clasificación de las herramientas había sido extraída de una primera recogida de información que se realizó de forma individual a los mismos estudiantes.

4 Se ha tomado como referencia los alumnos matriculados en las troncales y obligatorias puesto que son las asignaturas que tienen que cursar todos los alumnos.

llo curricular. En Herrán Gascón, Agustín y Paredes, Joaquín (coords.): Didáctica General. Madrid, McGrawhill.

Martínez Valcárcel, Nicolás (1998): Los problemas de especialización y globalización en los currícula de primaria. Notas para un debate. En Gómez, C. y Fernández, M. (Eds): La Función Docente en Educación Infantil y Primaria desde las nuevas especialidades. Granada, Grupo Editorial Universitario.

Marzano, Robert J. y Kendall, John S. (2007): The New Taxonomy of Educational Objectives. USA, Corwin Press.

Nieto Cano, José Miguel y Vallejo, Mónica (2010): Trabajos monográficos. En Porto Currás, Mónica (coord.): Técnicas de evaluación en el Espacio Europeo de Educación Superior: ejemplos prácticos. Murcia, Editum.

Porto Currás, Mónica (2006): La evaluación de estudiantes universitarios vista por sus protagonistas. Educatio Siglo XXI, no 24 , pp. 167-187.
5 El número total de asignaturas ofertadas para esta titulación, concretamente en este periodo, es de 120 asignaturas de las cuales los alumnos cursaron 93. Decimos que hemos llegado a todas las asignaturas cursadas, ya que las demás ofertadas no tuvieron alumnos matriculados en esta promoción.
Porto Currás, Mónica (2009): Evaluación de estudiantes en la Universidad de Santiago de Compostela: percepciones de los propios alumnos. Red U-Revista de Docencia Universitaria, 3. [Fecha de Consulta: 04/05/2010]. http://www.um.es/ead/Red_U/3/

Rodríguez Diéguez, José Luis (1980): Didáctica General. Objetivos y evaluación. Madrid, Cincel.

Salkind, Neil J. (1999): Métodos de investigación. México, Prentice Hall.

Velilla Barquero, Ricardo (1979): Como se realiza un trabajo monográfico. BarceIona, Eunibar.

Zabalza Beraza, Miguel Ángel (1991): Los diarios de clase. Barcelona, PPU.

Zabalza Beraza, Miguel Ángel (2003): Competencias docentes del profesorado universitario. Calidad y desarrollo profesional. Madrid: Narcea. 